\title{
Curriculum Sequencing for an e-learning System sased on Learning Styles
}

\author{
Clara-I Peña* · Jose-L Marzo Josep-Li de la Rosa \\ University of Girona, Spain \\ \{clarenes,marzo,peplluis\}@eia.udg.es '
}

\begin{abstract}
This paper shows the use of adaptation techniques involved in an e-learning system that considers students' learning styles and students' knowledge states. The mentioned $\mathrm{e}$ learning system is built on a multiagent framework designed to examine opportunities to improve the teaching and to motivate the students to learn what they want in a user-friendly and assisted environment.
\end{abstract}

\section{INTRODUCTION}

This work assumes that teachers should consider the cognitive styles of students in order to deliver adaptive education. In this sense, the system aims to construct a warehouse of courses in which each set of concepts, or ideas is affected by certain parameters chosen by the author's methodological criteria. These parameters can be classified into two groups: the first, which gives importance to the content (accumulation of knowledge - rigid pedagogy); and the second, which cedes a place to the chosen processes (construction of the knowledge, mediation, etc. - flexible pedagogy). This last group composes the heart of the MASPLANG' (see [4]), an adaptive multiagent hypermedia system for e-learning developed at the University of Girona, Spain. In this context, intelligent agents introduce a new paradigm for instruction since it is focused on the concept of shared abilities and cooperative learning between human and computers [5]. In Artificial Intelligence methodologies, the intelligence may be applied through user models to make assumptions about the user's state of knowledge, which may in turn help determine the user's learning needs [2]. MASPLANG agents are designed to improve the guidance giving to the user, while increasing the user motivation and the adaptivity of course materials. Choosing the right instructional strategy based on learning styles and providing access to didactic materials adapted to learning styles and levels of knowledge are two important aspects considered in this study.

This paper is organized as follows: section II presents the MASPLANG architecture designed for adaptive e-learning enhancing student assistance and student motivation. The

\footnotetext{
1 MASPLANG: Multiagent System PLANG. PLAANG was a project supported by the Spanish Research Council (CICYT) TEL 98-0408-C02-01 and TEL99-0976. Its name belongs from the Spanish sentence PLAtaforma de Nueva Generación'.
}

conceptual model of MASPLANG is described in section III. Conclusions are presented in section IV.

$$
\begin{aligned}
& \text { II. MASPLANG ARCHITECIURE FOR ADAPTIVE E- } \\
& \text { LEARNING }
\end{aligned}
$$

MASPLANG was developed with the purpose of giving adaptive characteristics, to a Course Management System named USD² (see [10]). It was designed using a two-level agent architecture (Information and Assistant agents), as shown in Fig. 1.

Assistant agents assist students as they work with the didactic material arranged for the course. Such assistance consists of registering the student actions (by, means of the Monitor agents) to identify patterns that can be used for personalizing the presentation of the learning content and the navigation tools the students use to move through the contents (by means of the Browsing agent) and to adapt exercises (by means of the Exercise Adapter agent) for self-assessment to the student's knowledge level or preferences. To make the student feel comfortable when he/she carries out the learning activities, an animated, lifelike character (the SMIT agent) has been designed to display the reinforcement information and the programmed alert messages (by means of the SONIA agent).

There are two Information agents. The first is the User agent designed to maintain the student model and the second is the Pedagogic agent which evaluates the pedagogic decision rules that are embedded in the pedagogic model of the course. The Information agents are very close to the system databases (students' learning activities dossier and domain model).

The services that MASPLANG agents offer to the elearning environment are summarized in Table 1. 


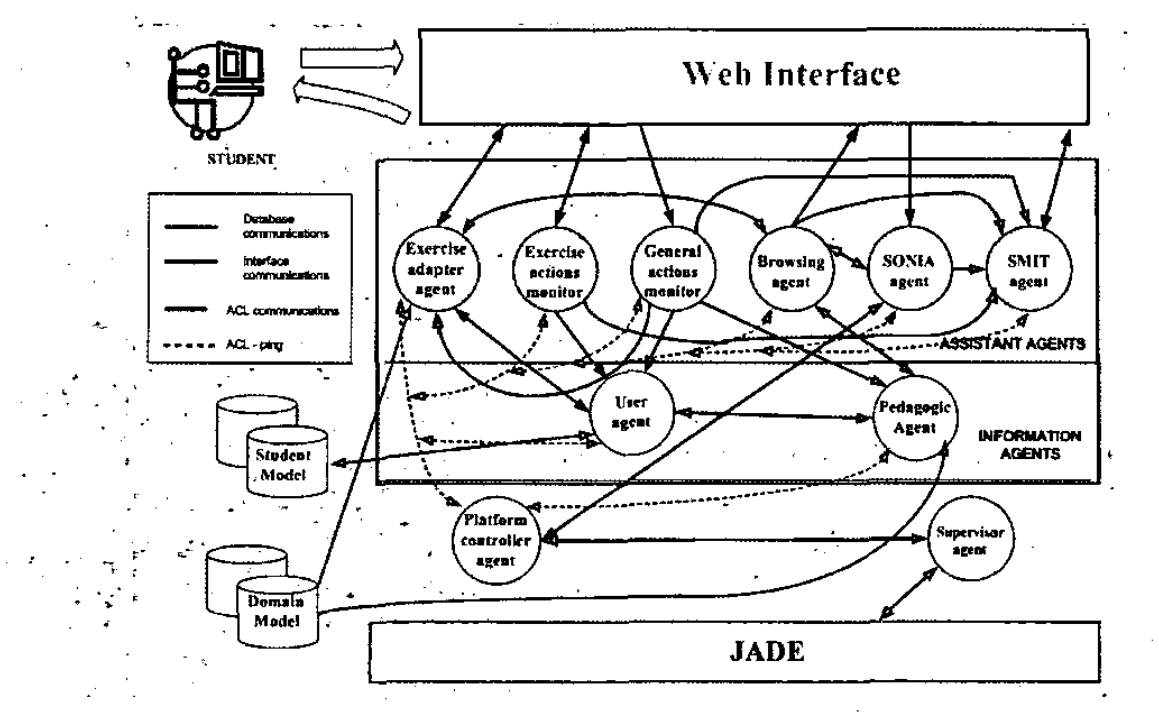

Fig. 1. MASPLANG two level agent architecture

TABLE I

MASPLANG ÁGENTS' SERVICES

\begin{tabular}{|c|c|}
\hline MASPLANG AGENT: & SERVICES \\
\hline $\begin{array}{l}\text { SMIT (Synthetic Multimedia } \\
\text { Interactive Tutor) }\end{array}$ & $\begin{array}{l}\text { Shows messages to the user via a life-like animated character } \\
\text { Interacts with the student when he/she wants to recall the message history that it has shown. }\end{array}$ \\
\hline $\begin{array}{l}\text { SONIA (Student Oriented } \\
\text { Network Interface Agent) }\end{array}$ & $\begin{array}{l}\text { Receives instructions from the user regarding the personalized tasks to be cartied out (reminders. } \\
\text { connected users search, alerts for the existence of bibliography or exercises in the lesson, etc.) } \\
\text { Asks the Controller agent to execute programmed tasks concerning the system's events } \\
\text { Asks the Browsing agent to execute programmed tasks concerming particular aspects of the } \\
\text { learning materials (links to bibliographical references or exercises) }\end{array}$ \\
\hline CONTROLLER & $\begin{array}{l}\text { Supervises the lifecycle of all the MASPLANG agents (using testing by means of ACL ping } \\
\text { messages) } \\
\text { Carries out tasks concerned with system events (especially those tasks programmed by means of } \\
\text { the SONIA agent, such as alarm clocks, users connections, etc.) }\end{array}$ \\
\hline $\begin{array}{l}\text { GENERAL ACTIONS } \\
\text { MONITOR }\end{array}$ & $\begin{array}{l}\text { Monitors the general user's actions } \\
\text { Requests the Exercise adapter agent to build an adapted exercise for the student if the student } \\
\text { clicks on the Exercise adapter agent button of the interface } \\
\text { Updates the knowledge base of the user agent }\end{array}$ \\
\hline EXERCISES' MONITOR & $\begin{array}{l}\text { Monitors the user's actions during exercises (collecting the student's answers) } \\
\text { Updates the knowledge base of the user agent } \\
\text { Sends feedback information to SMIT agent }\end{array}$ \\
\hline EXERCISE ADAPTER & $\begin{array}{l}\text { Builds an adapted exercise for the student, based on his/her exercise history at the User agent's } \\
\text { request or based on the default parameters at the Pedagogic agent's request }\end{array}$ \\
\hline SUPERVISOR & $\begin{array}{l}\text { Maintains a list of the users connected to the platform (extension of the JADE DF agent) } \\
\text { Sends a messatge to the Controller agent when a particular user is connected to the platform }\end{array}$ \\
\hline USER & $\begin{array}{l}\text { Models the student during session (maintains a temporary student model) } \\
\text { Updates the permanent student model } \\
\text { Responds to Pedagogic asent and Exercise adapter agent petitions }\end{array}$ \\
\hline BROWSING & $\begin{array}{l}\text { Receives the adapted learning information from the Pedagogic agent : } \\
\text { Adapts navigation paths in the student interface } \\
\text { Adapts navigation tools in the student interface } \\
\text { Manages the motivation information }\end{array}$ \\
\hline PEDAGOGIC & $\begin{array}{l}\text { Evaluates the pedagogic decision rules of the pedagogic domain } \\
\text { Asks the User agent for relevant information about the user included in rules } \\
\text { Adapts contents presentation }\end{array}$ \\
\hline
\end{tabular}




\section{HI. MASPLANG CONCEPTUAL MODEL For AdAPTIVE E- LEARNING}

The conceptual model of this system is composed basically of three models (see Fig. 2): the domain model that determines. the concepts to be taught and their interrelationships to provide a global structure of the concerned domain (knowledge domain); the student model that allows the student's different : features (i.e. expertise, knowledge, preferences, objectives, etc.) to be considered in the learning process; and the interaction, model that encapsulates the adaptive engine to offer adaptive presentation and adaptive navigation (see [9]) by means of supervising the student interaction.

The next formulation represents an abstract model of the MASPLANG performance considering the conceptual issues described above:

$$
\begin{array}{ll}
\text { IS: } & \mathrm{S} \leftarrow \mathrm{LP} \\
\mathrm{D}_{\mathrm{ks}}: & \mathrm{C} * \mathrm{O} \rightarrow \sum_{i=1}^{n} H T M L \\
\mathrm{ES}: & \mathrm{SM}_{\mathrm{S}} \leftarrow \mathrm{LP}_{\mathrm{ls}} * \mathrm{~K}_{\mathrm{S}}\left(\mathrm{D}_{\mathrm{k}}\right)
\end{array}
$$

Where:

$I S$ as in (1) means that at the beginning of the session a learning profile based on learning styles $\left(L P_{k s}\right)$ is assigned to the student $S$. The first value of this learning profile is obtained by the evaluation of the ILS (Index of Learning Style) questionnaire [8] applied to the student. ILS is the learning style diagnostic instrument of the FSLSM model Felder and Silverman Learning Style Model [7] - adopted for this study. Later on and after collecting a representative number of student actions, this profile is fine-tuned through a case-based reasoning process [6].

$D_{t s}$ as in (2) defines the knowledge domain model based on learning styles. It is composed by a set of contents (concepts) with an organization structure $(O)$. At the end, what the student receives from this domain model is a set of. HMTL pages.

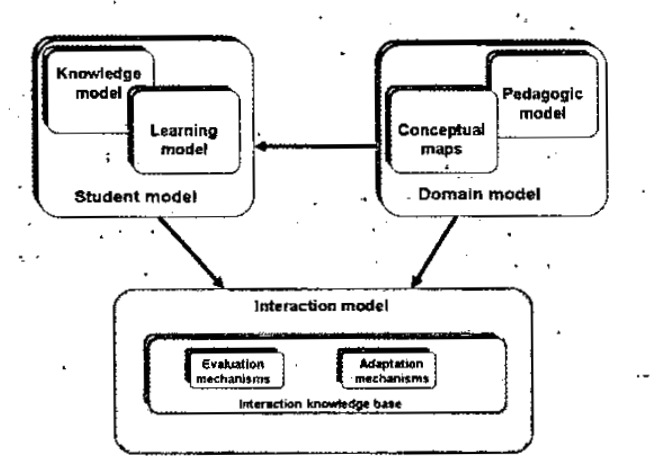

Fig. 2. MASPLANG Conceptual Model
Finally, ES as in (3) means that at the end of the learning session, the student model $\left(S M_{s}\right)$ is updated considering the student learning profile $\left(L P_{t s}\right)$ and the student knowledge state $\left(K_{S}\right)$.

\section{A. Domain Model}

The domain model, as part of an adaptive hypermedia system for education, represents both the knowledge about a particular domain that will be transmitted to the student and the way of presenting that information (rules defined in a pedagogic model).. The domain model knowledge and its structure determine the contents of the tutorial interaction, together with the structure that governs the adaptive instruction.

The domain model of the MASPLANG is declarative and its knowledge is represented by means of a conceptual map (see Fig. 3) whose-structure takes into account the static (the what) and the evolitionary (the how) focus.

From the static point of view, the teaching concepts are represented by means of a conceptual network structured using different taxonomies. Each node corresponds to a domain concept and it is disaggregated in other nodes using class-subclass relationships (i.e. tree like structure). The resultant conceptual network is a static representation of the knowledge in the teaching domain (i.e., what will be taught). :

From the evolutionary point of view, the conceptual network is structured using relationships to describe the pedagogic rules needed to. select the contents and/or determine their sequencing. In this study, conceptual (e.g. property relationships, such as " $\mathrm{X}$ is "part of $\mathrm{Y}$ ") and procedural relationships are considered.

The procedural relationships are used to determine the order in which the concept nodes should be learned or the decisions that should be evaluated to reach any instructional objective (e.g. if condition $\mathrm{A}$ is true then the student may study nodes 1.1 and 1.2 of the Concept 1 ). This structure corresponds to the didactic organization of the domain (i.e., how the concepts will be taught).

\section{B. Content Types that Consider Learning Styles}

Supporting quality teaching and learning has been one of the critical issues in distance education. In distance learning scenarios, one of the key things to consider is how the student feels about the educational material. This raises several critical issues concerning learning styles and dynamic pedagogic material adapted to particular student preferences.

Selecting the learning. style model is crucial to the development of an effective adaptive hypermedia course that addresses different learning style preferences. For this study, the FSLSM learning style model was adopted, since it has been well tested in web-delivered courseware for Engineering and Computer Science education (our field- of interest). 


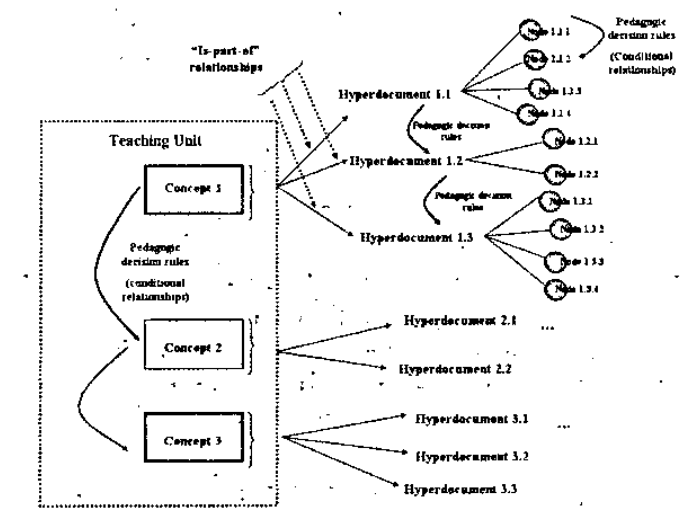

Fig. 3. Example of the MASPLANG Donain Model Organization

The experiences of Carver [3] using this model, have demonstrated that students are empowered to learn using their own unique learning style instead of being forced to learn according to the instructor's point of view.

Adapting some traditional instructional strategies and building the learning objects by means of HTML pages (since MASPLANG teachers have worked well developing contents in this format) which have subjects embedded in different media format, Table II offers a useful distribution of criteria for selecting instructional strategies, instructional complementary materials, interactive and assessment elements and navigation tools for adaptive presentation and adaptive navigation in MASPLANG using FSLSM learning styles.

TABLE II

Hypermedia Course COMPONENTS For MasPlang CONSidering FsLSM LEARNING S'TYLES
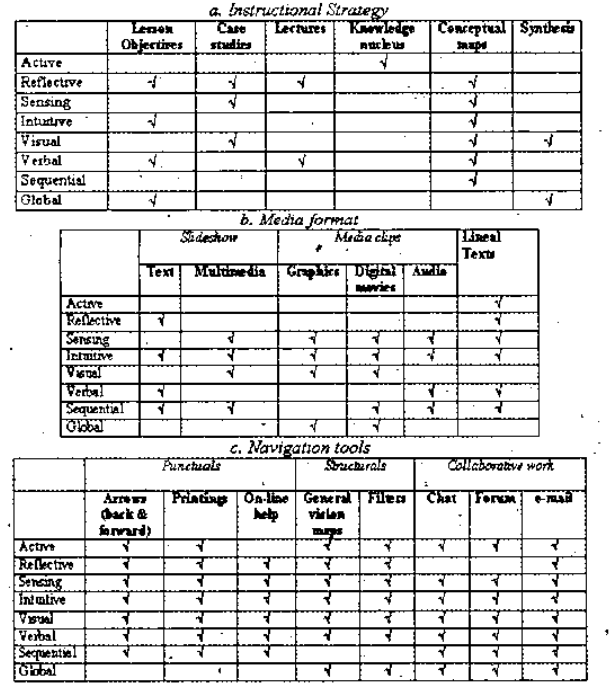

\section{Domain Model Building in MASPLANG}

As it was shown in Fig. 3, the MASPLANG domain model is represented by a semantic graph based on a set of concepts to teach. Each concept is considered as a basic learning unit with its own properties (e.g. associated learning style, required level of knowledge, requisites, etc.). Each concept also can be disaggregated in hyperdocuments and these in turn can be disaggregated in nodes. The relationships between concepts and hyperdocuments and between hyperdocuments and nodes are represented by links in the graph.

The graph may have different types of nodes and links as shown in Fig. 4. The main nodes should contain Basic information corresponding to theoretical explanations (node 1 in Fig. 4) or information for enforcing student assessment corresponding to exercises (node 4 in Fig. 4). Additionally, there are two optional types of nodes that may be associated to each main node: the bibliography nodes (node 2 in Fig. 4) that enable the bibliography review and the exercise nodes (node 4 in Fig. 4) that enable exercises for student self assessment (notice that exercise nodes may be of the main or optional types depending on how the nodes are linked in the graph). These nodes will be available when the main nodes are being studied.

There are six types of links as follows: the is-part-of link - link 1 in Fig. 4 - which connects the nodes that are part of a hyperdocument used to explain a concept or part of a concept. The bibliography link - link 2 in Fig. 4 - which points to an optional bibliography node (node 2 in the set of nodes of Fig. 4). This link may be enabled from any of the main nodes of the graph. The feedback link - link 3 of Fig. 4 - which points to a feedback information node (node 3 in the set of nodes of Fig. 4). This link may only be enabled in a decision link. The exercise link - link 4 in Fig. 4 - which points to an optional exercise node (node 4 in the set of nodes of Fig. 4). This link may be enabled from any of the main nodes of the graph. The sequence link - link 5 in Fig. 4 - which points to the next node to be visited, thus establishing a mandatory sequence. The decision link - link 6 in Fig. 4 -which allows the user to visit the next node once a particular condition is satisfied.

The information concerning exercises is the only one that is not prepared directly by teachers using HTML pages. Instead, teachers should build and store in the database (before making the graph), a global exercise skeleton with questions and answers (using the Exercise editor - a MASPLANG teaching. tool) organized by subjects and three levels of difficulty (1-easy, 2-normal and 3-difficult).

The exercise nodes referred in the semantic graph are built during the student learning session by the Exercise adapter agent in HTML format, using instances of the global exercise skeleton. At this point, the agent (that 


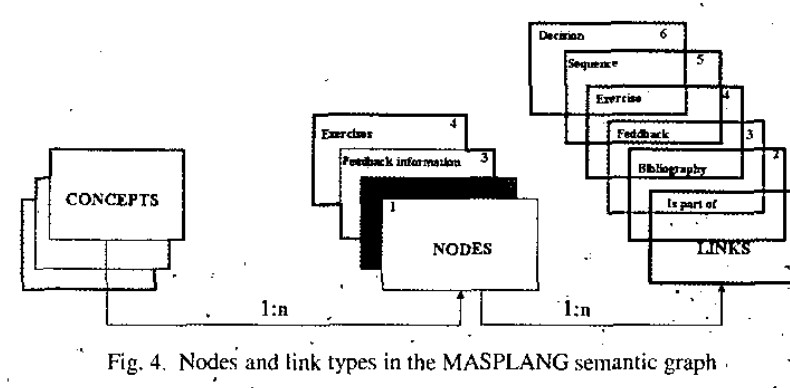

appears as an icon in the navigation tool bar) allows the student to decide if he/she wants to configure an instance of the exercise or to leave the agent to adapt the exercise according to the student knowledge state (applying principles of the Gagné theory [11]).

To build this domain model, the MASPLANG offers teachers the Teaching Units Editor that allows representing graphically the semantic graph. This work modality (i.e. drawing physically the graph) with the facilities afforded by the editor (i.e. an ergonomic and easy to use environment) has been well accepted by the MASPLANG teachers."

\section{Student Model}

A student model defines a knowledge base that establishes: the learning characteristics of a student; the knowledge that he/she has about the domain; the didactic material that he/she has used to learn; the history of the learning sessions that he/she has carried our; etc. This information is used by other components of the system to achieve a more efficient process of instruction which is better adapted to the student.

Two elements are taken into account when modeling the student in MASPLANG: the student model knowledge base (where the student learning characteristics are established) and the User agent (i.e., the student manager - an agent based on knowledge).

MASPLANG uses a hybrid model, a combination of an overlay model [1] and an inferred model, to represent the student knowledge about the domain. This model is in turn divided into two more conceptual models: one is permanent and the other is temporal.

The permanent model contains information concerned with data about the student's personal characteristics and his/her learning profile, the knowledge that he/she has abou the domain; the didactic material that he/she has used to learn and the history of the learning sessions carried out (e.g. common actions, history of exercises, etc.). This model is available during the whole instruction process and is updated session by session.

The first data acquired for the leaming profile comes from the ILS questionnaire (i.e. a task carried out by the general monitor agent of the multiagent architecture). Later, it is finetuned by analyzing the student's interactions with the system using a CBR approach that centers its process in determining the sensibility that a student or a group of students have to a teaching unit with particular attributes (e.g. media format for learning objects, teaching strategy, navigation tools, navigation techniques, etc.)

The knowledge about the domain is the knowledge that the student has acquired through the learning process and how this knowledge was acquired. The particular structure of the domain is modified to include new attributes to control the acquisition characteristics (i.e. the navigation through the graph is adapted to the student knowledge state). These attributes are: the acquired-level to indicate the level of knowledge that the student has about a concept and the concepts that the student has learnt.

The didactic material that the student has used to learn (i.e. basic contents and exercises) identifies the material used by the system to present the learning content or to assess it. This information is used by the pedagogic agent of the MASPLANG architecture to make a suitable choice of the content that the student should learn at any particular time. The exercise adapter agent uses the information concerned with the exercises already solved by the student to adapt exercises to the student knowledge state.

In the student model, the information about the development of the instruction process is also considered. Data about the last session summarizes the learning session events that took place in the last session. This information is important for setting up certain elements to be represented in the next session.

The history of the whole instructional process is represented by a set of actions commonly carried out, which nodes were visited along with the time spent on each visit and information about the student development when solving exercises (i.e. a list of the exercises that the student has made and the way they were solved).

The temporal model makes sense only for the current session. Its data is managed for the User agent who at the end of the session updates the permanent model with relevant information that should alter the student knowledge state.

\section{E. The Interaction model}

As it was shown in Table I that described the MASPLANG agent services, is the Pedagogic agent who defines the navigation paths and the contents that a student may study progressively in a learning session according to the student model and the structure of the domain. To carry 
out these adaptive tasks, the Pedagogic agent evaluates the decision rules of the pedagogic domain requesting suitable information about the student model from the User agent to build a navigation tree structure or a concept state diagram for the student at any moment (see in Fig. 5 the construction of strong and light links which delimit the suitable paths and nodes that the student may follow). The information that the student receives is presented latter by the Browsing agent through a personalized interface with ergonomic navigation tools and applying adaptive navigation techniques such as link hidden, direct guidance and link annotation [9].

Student assistance and student motivation messages are displayed by $S M I T$, a synthetic agent that is introduced in the environment using an animated interface (anthropomorphous). Its goal is to show the student the messages (e.g. warnings, motivation, feedback, etc.) coming from other agents in the environment. Each message representation demands the selection of certain animations and body movements to define the SMIT behavior in any particular situation. The aim of using this agent is "to "humanize" the learning environment and to make it user-friendlier and closer to the student. See in Fig. 6 an aspect of SMIT agent representing a multicast message coming from a teacher.

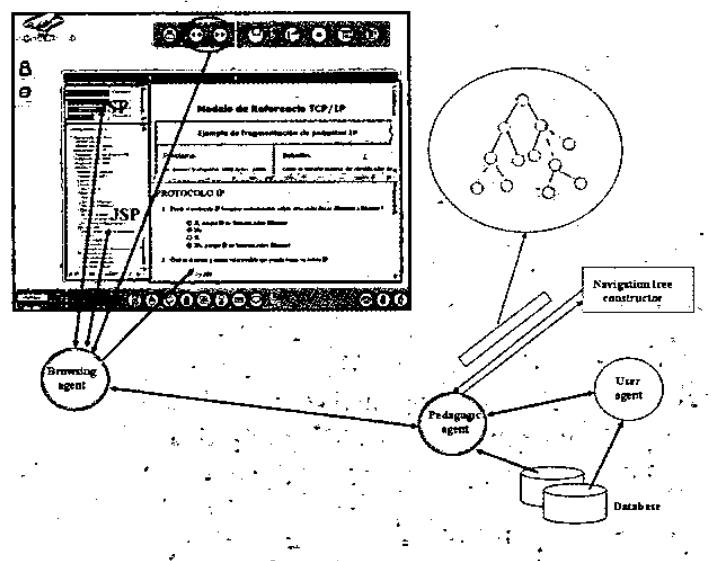

Fig. 5. Interaction activities between the Pedagogic, the User and, the Browsing agents to display an adaptive content

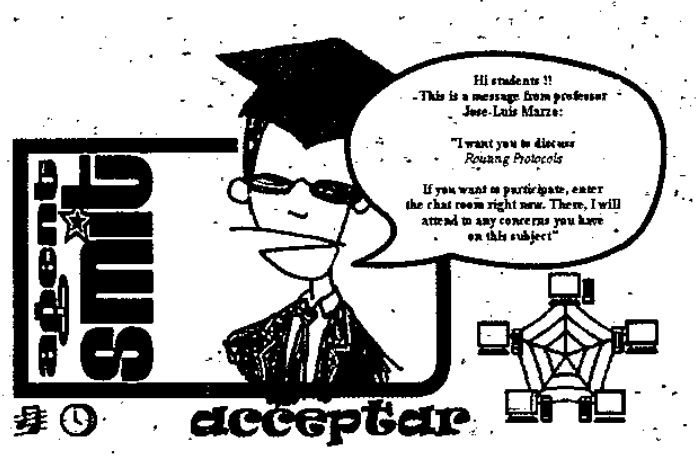

Fig. 6. The SMIT agent displaying a teacher's message to the studenit

\section{CONCLUSIONS}

In this work, some pedagogical and technological aspects concerning e-learning for a particular case have been presented. The main motivation in proposing the MASPLANG architecture for adaptive e-learning was the need to offer students the didactic material best suited to their individual learning profile. In achieving the proposed objectives of adaptivity, agent technology has been successfully applied. Working with agents has been highly interesting. Although the multiagent architecture seems quite complex, the parallelization of the work allowed by such technology was crucial to obtain the desired functionality in MASPLANG.

The conceptual model of the proposed adaptive hypermedia system is based on a standard architecture; however, the domain, student and interaction models have been designed and added on the basis of previous experience using a former platform (the USD).

The system makes it easier for teachers to design, publish and manage their courses. A set of high level, flexible and ergonomic tools are provided for creating the domain model, to check on student progress and to communicate with them.

\section{REFERENCES}

[1] B. Carr and 1. Goldstein, "Overlays: A theory of modeling for computer aided instruction". (AI Memo 406), Cambridge, MA: Massachusetts Institute of Technology, AI Laboratory, 1977.

[2] B. Woolf, "Intelligent muitimedia tutoring systems," Communications of the ACM, Vol. 39, No. 4, 1996, pp. 30-1.

[3] C.A. Carver, R.A. Howard, and W.D. Lane, "Addressing Different Learning Styles Through Course Hypernedia," IEEE Transactions on Education, 42(1), 1999, pp. 33-38.

[4] C.I. Peña, J.L. Marzo, and J.L. De la Rosa, "Intelligent Agents in a Teaching and Learning Environment on the Web," 2ond-IEEE International Conference. on Advanced Learning Technologies (ICALT2002), Kazan (Russia). September 9-12, 2002, pp. 21-27.

[5] G. Kearsley, "Intelligent agents and instructional systems: implications of a new paradigm," Journal of Artificial Intelligence in Education, Vol.. 4, No. 4, 1993. pp. 295-304.

[6] Habitat-Pro ${ }^{\text {TM }}$ Environment, Agents Inspired Technologies Corporation, University of Girona, Girona. Spain, 2001, http://www.agentsinspired.com.

[7] M. R. Felder and L. Silverman, "Learning and Teaching Styles in Engineering Education", In Engineering Education 78(7), 1988. pp. 674-681.

[8] M.R. Felder and L.- Silverman. "Diagnostic instrument for the FSLSM ". " model.," http:/www2.ncsu edu/unity/lockers/users/f/felder/public/lLSdir/ilis web.html.

-[9] P. Brusilovsky. "Methods and techniques of adaptive hypermedia." Journal of User Modeling and User Adapted Interaction, 6, (2-3). 1996, pp. 87-129.

[10] R. Fabregat, J:L. Marzo, C.I. Peña, "Teaching Support Units", Computers and Education in the 21 st Century, Kluwer Academic Publishers, 2000, pp. 163-174.

[11] R. Gagne, L. Briggs, L. \& W. Wager,. Principles of Instructional Design (4th Ed.). Fort Worth, TX: HBJ College Publishers, 1992. 